\title{
On the global log canonical threshold of Fano complete intersections
}

\author{
Thomas Eckl ${ }^{1}$ • Aleksandr Pukhlikov ${ }^{1}$
}

\begin{abstract}
We show that the global log canonical threshold of generic Fano complete intersections of index 1 and codimension $k$ in $\mathbb{P}^{M+k}$ is equal to 1 if $M \geqslant 3 k+4$ and the highest degree of defining equations is at least 8 . This improves the earlier result where the inequality $M \geqslant 4 k+1$ was required, so the class of Fano complete intersections covered by our theorem is considerably larger. The result implies, in particular, that the Fano complete intersections satisfying our assumptions admit a Kähler-Einstein metric. We also show the existence of Kähler-Einstein metrics for a new finite set of families of Fano complete intersections.
\end{abstract}

Keywords Fano variety $\cdot$ Birational rigidity $\cdot$ Kähler-Einstein metric $\cdot$ Hypertangent divisor

Mathematics Subject Classification $14 \mathrm{E} 05 \cdot 14 \mathrm{E} 07 \cdot 14 \mathrm{~J} 45$

\section{Canonical and log canonical thresholds}

Consider a smooth Fano variety $X$, such that Pic $X=\mathbb{Z} K_{X}, D \sim-n K_{X}$ an effective divisor, $n \geqslant 1$.

Aleksandr Pukhlikov

pukh@liv.ac.uk

Thomas Eckl

eckl@liv.ac.uk

1 Department of Mathematical Sciences, The University of Liverpool, Peach St.,

Liverpool L69 7ZL, UK 
Definition 1.1 The pair $(X, \alpha D)$, where $\alpha \in \mathbb{Q}$, is not canonical (respectively, not $\log$ (anonical), if there exists a prime divisor $E$ over $X$ such that the inequality

$$
\alpha \operatorname{ord}_{E} D>a(E)
$$

(respectively, $\left.\alpha \operatorname{ord}_{E} D>a(E)+1\right)$ is satisfied, where $a(E)=a(X, E)$ is the discrepancy of $E$ with respect to the model $X$.

Explicitly, this means that there are a birational morphism $\varphi: \widetilde{X} \rightarrow X$, where $\widetilde{X}$ is a smooth projective variety, and a prime $\varphi$-exceptional divisor $E \subset \widetilde{X}$ such that

$$
\alpha \operatorname{ord}_{E} \varphi^{*} D>a(E)
$$

(respectively, $\left.\alpha \operatorname{ord}_{E} \varphi^{*} D>a(E)+1\right)$.

Definition 1.2 The global canonical (respectively, log canonical) threshold of the variety $X$ is defined by the equality

$$
\operatorname{ct}(X)=\sup \left\{\lambda \in \mathbb{Q}_{+}:\left(X, \frac{\lambda}{n} D\right) \text { is canonical for all } D \in\left|-n K_{X}\right|\right\},
$$

respectively,

$$
\operatorname{lct}(X)=\sup \left\{\lambda \in \mathbb{Q}_{+}:\left(X, \frac{\lambda}{n} D\right) \text { is } \log \text { canonical for all } D \in\left|-n K_{X}\right|\right\} .
$$

(Note that as $D \sim-n K_{X}$, the integer $n \geqslant 1$ depends on the effective divisor $D$.)

The importance of canonical and log canonical thresholds comes from their applications to complex differential geometry and to certain problems of higher-dimensional birational geometry.

In $[1,2,9]$ the following fact was shown.

Theorem 1.3 Assume that the inequality

$$
\operatorname{lct}(X)>\frac{\operatorname{dim} X}{\operatorname{dim} X+1}
$$

holds. Then on the variety $X$ there exists a Kähler-Einstein metric.

The log canonical threshold is important in this differential-geometric context because it indicates the non-triviality of certain multiplier ideal sheaves. In their analytic interpretation, these multiplier ideal sheaves in turn measure the failure of a priori estimates sufficient to solve a Monge-Ampère equation for a Kähler-Einstein metric.

Definition 1.4 The mobile canonical threshold of a variety $X$, which is denoted by $\operatorname{mct}(X)$, is the supremum of $\lambda \in \mathbb{Q}_{+}$such that the pair $(X, \lambda D / n)$ is canonical for a generic divisor $D \in \Sigma$ of any mobile linear system $\Sigma \subset\left|-n K_{X}\right|$ (that is to say, any system $\Sigma$ with no fixed components). 
In [5] the following fact was shown.

Theorem 1.5 Assume that primitive Fano varieties $F_{1}, \ldots, F_{K}, K \geqslant 2$, satisfy the conditions $\operatorname{lct}\left(F_{i}\right)=\operatorname{mct}\left(F_{i}\right)=1$. Then their direct product

$$
V=F_{1} \times \cdots \times F_{K}
$$

is a birationally superrigid variety. In particular,

(i) Every structure of a rationally connected fiber space on the variety $V$ is given by a projection onto a direct factor. More precisely, let $\beta: V^{\#} \rightarrow S^{\#}$ be a rationally connected fiber space and $\chi: V \rightarrow V^{\#}$ a birational map. Then there exists a subset of indices $I=\left\{i_{1}, \ldots, i_{k}\right\} \subset\{1, \ldots, K\}$ and a birational map

$$
\alpha: F_{I}=\prod_{i \in I} F_{i} \rightarrow S^{\#},
$$

such that the diagram

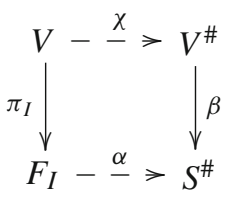

commutes, that is, $\beta \circ \chi=\alpha \circ \pi_{I}$, where

$$
\pi_{I}: \prod_{i=1}^{K} F_{i} \rightarrow \prod_{i \in I} F_{i}
$$

is the natural projection onto a direct factor.

(ii) Let $V^{\#}$ be a variety with $\mathbb{Q}$-factorial terminal singularities, satisfying the condition

$$
\operatorname{dim}_{\mathbb{Q}}\left(\operatorname{Pic} V^{\#} \otimes \mathbb{Q}\right) \leqslant K
$$

and $\chi: V \rightarrow V^{\#}$ a birational map. Then $\chi$ is a (biregular) isomorphism.

(iii) The groups of birational and biregular self-maps of the variety $V$ coincide:

$$
\text { Bir } V=\text { Aut } V \text {. }
$$

In particular, the group $\operatorname{Bir} V$ is finite.

(iv) The variety $V$ admits no structures of a fibration into rationally connected varieties of dimension strictly smaller than $\min \left\{\operatorname{dim} F_{i}\right\}$. In particular, $V$ admits no structures of a conic bundle or a fibration into rational surfaces.

(v) The variety $V$ is non-rational.

For the precise definition of birational (super)rigidity, a discussion of its properties and examples of birationally (super)rigid varieties, see [8]. 


\section{Fano complete intersections}

Fix an integer $k \geqslant 2$. Consider an arbitrary system $\left(d_{1}, \ldots, d_{k}\right)$ of positive integers satisfying the condition $d_{k} \geqslant \cdots \geqslant d_{1} \geqslant 2$. Set $M=d_{1}+\cdots+d_{k}-k$. Fix the complex projective space $\mathbb{P}=\mathbb{P}^{M+k}$ and consider the family $\mathcal{F}\left(d_{1}, \ldots, d_{k}\right)$ of non-singular complete intersections $V$ of the type $d_{1} \cdots d_{k}$ in $\mathbb{P}$ :

$$
V=F_{1} \cap \cdots \cap F_{k} \subset \mathbb{P}
$$

here $F_{i} \subset \mathbb{P}$ is a hypersurface of degree $d_{i}$, and codim $V=k$.

The following two theorems collect the known information about the global canonical and log canonical thresholds of Fano complete intersections as above.

Theorem 2.1 ([6, Section 3]) Assume that $M \geqslant 4 k+1$ and $d_{k} \geqslant 8$. Then for $a$ generic (in the sense of Zariski topology on the space $\mathcal{F}\left(d_{1}, \ldots, d_{k}\right)$ ) variety $V \in$ $\mathcal{F}\left(d_{1}, \ldots, d_{k}\right)$ the equality $\operatorname{ct}(V)=1$ holds.

Thus under the assumptions of Theorem 2.1 on $V$ there exists a Kähler-Einstein metric. Besides, since $\operatorname{lct}(V) \geqslant \operatorname{ct}(V)$ and $\operatorname{mct}(V) \geqslant \operatorname{ct}(V)$, the variety $V$ satisfies the assumptions of Theorem 1.5 and for that reason can be used as a direct factor in birationally superrigid Fano direct products.

Theorem 2.2 ([7]) Assume that $M \geqslant 4 k+1$ and any of the following conditions holds:

(i) $d_{k}=d_{k-1}=7$ and $M \leqslant 47$,

(ii) $d_{k}=7, d_{k-1} \leqslant 6$ and $M \leqslant 19$,

(iii) $k=2, d_{1}=d_{2}=6, M=10$.

Then the canonical threshold $\operatorname{ct}(V)$ (and hence also the log canonical threshold $\operatorname{lct}(V))$ of a generic variety $V \in \mathcal{F}\left(d_{1}, \ldots, d_{k}\right)$ satisfies the inequality

$$
\operatorname{ct}(V)>\frac{M}{M+1} \text {. }
$$

Therefore, on a general variety $V \in \mathcal{F}\left(d_{1}, \ldots, d_{k}\right)$, satisfying one of the conditions listed in Theorem 2.2, there exists a Kähler-Einstein metric.

The aim of the present note is to improve the claims of Theorems 2.1 and 2.2, extending them to a larger class of Fano complete intersections of index 1. We will show the following two facts.

Theorem 2.3 Assume that $M \geqslant 3 k+4$ and $d_{k} \geqslant 8$. Then for a generic (in the sense of Zariski topology on the space $\left.\mathcal{F}\left(d_{1}, \ldots, d_{k}\right)\right)$ variety $V \in \mathcal{F}\left(d_{1}, \ldots, d_{k}\right)$ the equality $\operatorname{ct}(V)=1$ holds.

Theorem 2.4 Assume that $M \geqslant 3 k+4$ and any of conditions (i)-(iii) of Theorem 2.2 holds. Then the canonical threshold $\mathrm{ct}(V)$ (and hence also the log canonical threshold $\operatorname{lct}(V))$ of a generic variety $V \in \mathcal{F}\left(d_{1}, \ldots, d_{k}\right)$ satisfies inequality (1). 
Remark 2.5 Theorem 2.3 covers a considerably larger class of Fano complete intersections than Theorem 2.1. The same is true for the part (i) of Theorems 2.4 and 2.2. For the part (iii), Theorem 2.4 gives nothing new compared to Theorem 2.2. As for the part (ii), Theorem 2.4 gives the existence of the Kähler-Einstein metric for the following seven families of Fano complete intersections that do not fit into the assumptions of Theorem 2.2, all of them are in $\mathbb{P}^{24}$ :

$$
\begin{array}{llll}
\mathcal{F}(2,5,5,5,7), & \mathcal{F}(2,4,5,6,7), & \mathcal{F}(2,3,6,6,7), & \mathcal{F}(3,3,5,6,7), \\
\mathcal{F}(3,4,5,5,7), & \mathcal{F}(3,4,4,6,7), & \mathcal{F}(4,4,4,5,7) . &
\end{array}
$$

\section{Conditions of general position}

Now we will explain what we mean by the genericity of a Fano complete intersection $V \in \mathcal{F}\left(d_{1}, \ldots, d_{k}\right)$. Fix any point $o \in V$ and let $\left(z_{1}, \ldots, z_{M+k}\right)$ be a system of affine coordinates on $\mathbb{P}$ with the origin at the point $o$,

$$
f_{i}=q_{i, 1}+\cdots+q_{i, d_{i}}
$$

the equation of the hypersurface $F_{i}$ with respect to (non-homogeneous) coordinates $z_{*}$, decomposed into homogeneous in $z_{*}$ components $q_{i, j}, \operatorname{deg} q_{i, j}=j$. Since $V$ is a non-singular variety, the system of linear equations

$$
q_{1,1}=\cdots=q_{k, 1}=0
$$

defines a linear subspace $T_{o} V \subset \mathbb{C}^{M+k}$ of codimension $k$, the tangent space to the variety $V$ at the point $o$. We define a finite set of pairs $I \subset \mathbb{Z}_{+} \times \mathbb{Z}_{+}$in the following way: if $d_{k-1}=d_{k}$, then we set

$$
I=\left\{(i, j): 1 \leqslant i \leqslant k, 1 \leqslant j \leqslant d_{i},(i, j) \notin\left\{\left(k, d_{k}\right),\left(k-1, d_{k-1}\right)\right\}\right\},
$$

and if $d_{k-1} \leqslant d_{k}-1$, then we set

$$
I=\left\{(i, j): 1 \leqslant i \leqslant k, 1 \leqslant j \leqslant d_{i},(i, j) \notin\left\{\left(k, d_{k}\right),\left(k, d_{k}-1\right)\right\}\right\} .
$$

Definition 3.1 We say that the complete intersection $V$ is regular at the point $o$, if for any linear form $l\left(z_{*}\right)$, not vanishing identically on the subspace $T_{o} V$, the set of homogeneous polynomials

$$
\{l\} \cup\left\{q_{i, j}:(i, j) \in I\right\}
$$

forms a regular sequence in $\mathcal{O}_{o, \mathbb{P}}$, that is, the system of equations in $\mathbb{C}^{M+k}$

$$
l=0, \quad q_{i, j}=0, \quad(i, j) \in I,
$$

defines a subset of codimension \# $I+1$. Finally, we say that the complete intersection $V$ is regular, if it is regular at every point. 
In Sect. 4 we show the following fact.

Theorem 3.2 For $M \geqslant 3 k+4$ there exists a non-empty Zariski open subset

$$
\mathcal{F}_{\text {reg }}\left(d_{1}, \ldots, d_{k}\right) \subset \mathcal{F}\left(d_{1}, \ldots, d_{k}\right)
$$

such that any variety $V \in \mathcal{F}_{\mathrm{reg}}\left(d_{1}, \ldots, d_{k}\right)$ is regular.

By genericity in Theorems 2.1-2.4 we mean the regularity. For that reason, the main results of this paper (Theorems $2.3,2.4$ ) are essentially dependent on Theorem 3.2 which allows us to assume regularity of the complete intersection $V$.

\section{Proof of regularity conditions}

The proof of Theorem 3.2 proceeds in a series of reduction steps and case distinctions, through estimates (2)-(13).

Let the incidence variety $\mathcal{V} \subset H^{0}\left(\mathbb{P}^{M+k}, \mathcal{O}_{\mathbb{P}^{M+k}}(1)\right) \times \mathbb{P}^{M+k} \times \mathcal{F}\left(d_{1}, \ldots, d_{k}\right)$ consist of tuples $\left(L, o, F_{1}, \ldots, F_{k}\right)$ such that $o \in\left\{L=F_{1}=\cdots=F_{k}=0\right\}$. Let $p, q, r$ be natural projections of $\mathcal{V}$ to $H^{0}\left(\mathbb{P}^{M+k}, \mathcal{O}_{\mathbb{P}^{M+k}}(1)\right), \mathbb{P}^{M+k} \operatorname{resp.} \mathcal{F}\left(d_{1}, \ldots, d_{k}\right)$.

The image of $\mathcal{V}$ under the projection $p \times q$ is the incidence variety

$$
\mathcal{L} \subset H^{0}\left(\mathbb{P}^{M+k}, \mathcal{O}_{\mathbb{P}^{M+k}}(1)\right) \times \mathbb{P}^{M+k}
$$

consisting of pairs $(L, o)$ such that $L(o)=0$. All $(p \times q)$-fibers $\mathcal{V}_{L, o} \subset \mathcal{V}$ over points $(L, o) \in \mathcal{L}$ are isomorphic to a product of affine spaces and have codimension $k$ in $\mathcal{F}\left(d_{1}, \ldots, d_{k}\right)$, as vanishing in $o$ imposes one linear condition on the sections in $H^{0}\left(\mathbb{P}^{N}, \mathcal{O}_{\mathbb{P}^{N}}\left(d_{i}\right)\right)$. Hence $\mathcal{V}$ is irreducible.

Bertini's theorem shows that for a general tuple $\left(F_{1}, \ldots, F_{k}\right) \in \mathcal{F}\left(d_{1}, \ldots, d_{k}\right)$ the algebraic subset $\left\{F_{1}=\cdots=F_{k}=0\right\}$ is an $M$-dimensional complete intersection. Hence the general $r$-fiber in $\mathcal{V}$ has dimension $(M+k)+M=2 M+k$.

Let $\mathcal{V}^{\text {sm,nonreg }}$ be the locally Zariski-closed subset of tuples $\left(L, o, F_{1}, \ldots, F_{k}\right) \in \mathcal{V}$ such that $V=\left\{F_{1}=\cdots=F_{k}=0\right\}$ is a smooth complete intersection of dimension $M$, but the dehomogenisation $l$ of the linear form $L$ in affine coordinates around $o$ and the homogeneous parts $q_{i, j}$ of the dehomogenized $F_{i}$ do not satisfy the regularity condition of Definition 3.1. Then the theorem is shown if the projection of the Zariskiclosure of $\mathcal{V}^{\text {sm, nonreg }}$ does not cover $\mathcal{F}\left(d_{1}, \ldots, d_{k}\right)$.

To show this claim we note that every $r$-fiber of $V^{\text {sm,nonreg }}$ must be at least $k$ dimensional: If the subscheme $V=\left\{F_{1}=\cdots=F_{k}=0\right\} \subset \mathbb{P}^{M+k}$ is smooth of dimension $M$ in $o \in \mathbb{P}^{M+k}$ then we can choose homogeneous coordinates $\left[Z_{0}: \ldots\right.$ : $\left.Z_{M+k}\right]$ on $\mathbb{P}^{M+k}$ such that $o=[1: 0: \ldots: 0]$ and $q_{1,1}=z_{M+1}, \ldots, q_{k, 1}=z_{M+k}$ in the affine coordinates $z_{1}, \ldots, z_{M+k}$ dehomogenized with respect to $Z_{0}$. Hence there is a $k$-dimensional linear subspace of linear forms $L \in H^{0}\left(\mathbb{P}^{M+k}, \mathcal{O}_{\mathbb{P}^{M+k}}(1)\right)$ with the same intersection of $\{L=0\} \subset \mathbb{P}^{M+k}$ and the tangent space $T_{o} V$, seen as a linear subspace of $\mathbb{P}^{M+k}$. In particular, if the sequence $l, q_{1,1}, \ldots, q_{k, 1}, q_{1,2}, \ldots$ is not regular for one such linear form $L$ (dehomogenized to $l$ ) then the sequence will not be 
regular for any other linear form in this $k$-dimensional linear subspace. Consequently, we only need to show that

$$
\operatorname{codim}_{\mathcal{V}} \mathcal{V}^{\text {sm, nonreg }} \geqslant(2 M+k)-k+1=2 M+1 .
$$

The $q$-fibers $\mathcal{V}_{o}$ over points $o \in \mathbb{P}^{M+k}$ are all isomorphic. Consequently it is enough to show for all subsets $\mathcal{V}^{\text {sm, nonreg }} \cap \mathcal{V}_{o}=\mathcal{V}_{o}^{\text {sm, nonreg }}$ locally Zariski-closed in $\mathcal{V}_{o}$ that

$$
\operatorname{codim} \mathcal{V}_{o} \mathcal{V}_{o}^{\text {sm,nonreg }} \geqslant 2 M+1
$$

Let $\mathcal{P}_{d}^{N}$ denote the vector space of homogeneous polynomials of degree $d$ in the affine coordinates $z_{1}, \ldots, z_{N}, N \leqslant M+k$, introduced above. Then

$$
\mathcal{V}_{o} \cong \mathcal{P}_{1}^{M+k} \times \prod_{i=1}^{k} \prod_{j=1}^{d_{i}} \mathcal{P}_{j}^{M+k}
$$

by identifying the dehomogenized sections in $H^{0}\left(\mathbb{P}^{M+k}, \mathcal{O}_{\mathbb{P}^{M+k}}(j)\right)$ vanishing in $o$ with $\mathcal{P}_{j}^{M+k}$ and decomposing the dehomogenisation $f_{i}=q_{i, 1}+\cdots+q_{i, d_{i}}$ of $F_{i}$ in the tuple $\left(L, F_{1}, \ldots, F_{k}\right) \in \mathcal{V}_{o}$ into homogeneous parts $q_{i, j}$ of degree $j$.

Consider the projection $s$ of $\mathcal{V}_{o}$ to $\mathcal{P}_{1}^{M+k} \times \prod_{i=1}^{k}\left(\mathcal{P}_{1}^{M+k}\right)$, that is, to the linear form $l$ coming from $L$ and the linear parts $q_{i, 1}$ of dehomogenized $F_{i}$. The map $s$ is not defined everywhere on $V_{o}$ but since $V_{o}^{\text {sm,nonreg }}$ consists of tuples $\left(L, F_{1}, \ldots, F_{k}\right)$ such that $V=\left\{F_{1}=\cdots=F_{k}=0\right\} \subset \mathbb{P}^{M+k}$ is smooth of codimension $k$ in $o$ and $l_{\mid T_{o} V} \not \equiv 0$, hence none of $l$ and $q_{i, 1}$ can be 0 , we conclude that $s$ is defined on $\mathcal{V}_{o}^{\text {sm,nonreg }}$.

Therefore, it is enough to show that the codimension of the isomorphic $s$-fibers in $\mathcal{V}_{o}^{\text {sm,nonreg }}$ is $\geqslant 2 M+1$. Choosing the coordinates $z_{*}$ such that $l=z_{M}, q_{i, 1}=$ $z_{M+1}, \ldots, q_{k, 1}=z_{M+k}$, that means to show that the set $U\left(d_{1}, \ldots, d_{k}\right)$ of tuples of homogeneous polynomials in variables $z_{1}, \ldots, z_{M-1}$ defined by

$$
\left\{\left(q_{i, j}\right)_{1 \leqslant i \leqslant k, 2 \leqslant j \leqslant d_{i}}:\left(q_{i, j}\right)_{(i, j) \in I, j \neq 1} \text { is not a regular sequence in } \mathcal{O}_{0, \mathbb{A}}{ }^{M-1}\right\}
$$

and Zariski-closed in $\prod_{i=1}^{k} \prod_{j=2}^{d_{i}} \mathcal{P}_{j}^{M-1}$ satisfies

$$
\operatorname{codim}_{\prod_{i=1}^{k} \prod_{j=2}^{d_{i}} \mathcal{P}_{j}^{M-1}} U\left(d_{1}, \ldots, d_{k}\right) \geqslant 2 M+1 .
$$

In order to obtain this estimate only the degrees of homogeneous polynomials $q_{i, j}$ matter. So we will discuss the codimension of the set of tuples

$$
U=\left\{\left(q_{i}\right)_{1 \leqslant i \leqslant M}:\left(q_{i}\right)_{1 \leqslant i \leqslant M-2} \text { is not a regular sequence in } \mathcal{O}_{0, \mathbb{A}^{M-1}}\right\}
$$


in $\prod_{i=1}^{M} \mathcal{P}_{m_{i}}^{M-1}$, where $2 \leqslant m_{1} \leqslant \cdots \leqslant m_{M}=d_{k}$, and we have

$$
k_{d}=\#\left\{d_{l}: d_{l} \geqslant d, 1 \leqslant l \leqslant k\right\}
$$

polynomials $q_{i}$ of degree $d$. In particular $k=k_{2} \geqslant k_{3} \geqslant \cdots \geqslant k_{d_{k}}$ and

$$
\sum_{i=1}^{M} m_{i}=\sum_{d=2}^{d_{k}} k_{d} \cdot d=\sum_{l=1}^{k} \sum_{d=2}^{d_{l}} d=\sum_{l=1}^{k} \frac{d_{l}\left(d_{l}+1\right)}{2}-k
$$

Let $Z\left(q_{1}, \ldots, q_{j}\right) \subset \mathbb{A}^{M-1}$ denote the vanishing locus of $q_{1}, \ldots, q_{j}$ in $\mathbb{A}^{M-1}$. Since $q_{i}$ are homogeneous, $Z\left(q_{1}, \ldots, q_{j}\right)$ is a cone over the origin in $\mathbb{A}^{M-1}$, and we denote its projectivization in $\mathbb{P}^{M-2}$ by $V\left(q_{1}, \ldots, q_{j}\right)$. In particular, $\left(q_{1}, \ldots, q_{j}\right)$ is regular in $0 \in \mathbb{A}^{M-1}$ if and only if

$$
\operatorname{codim}_{\mathbb{P}^{M-2}} V\left(q_{1}, \ldots, q_{j}\right)=\operatorname{codim}_{\mathbb{A}^{M-2}} Z\left(q_{1}, \ldots, q_{j}\right)=j
$$

where the codimension is set to be the minimum of codimensions of the irreducible components.

Consequently, $U$ is covered by locally Zariski-closed subsets $U_{j} \times \prod_{i=j+1}^{M} \mathcal{P}_{m_{i}}^{M-1}$, where $1 \leqslant j \leqslant M-2$ and

$$
\begin{aligned}
U_{j}=\left\{\left(q_{1}, \ldots, q_{j}\right): \operatorname{codim}_{\mathbb{P}^{M-2}}\right. & V\left(q_{1}, \ldots, q_{j}\right) \\
& \left.=\operatorname{codim}_{\mathbb{P}^{M-2}} V\left(q_{1}, \ldots, q_{j-1}\right)=j-1\right\}
\end{aligned}
$$

is a locally Zariski-closed subset of $\prod_{i=1}^{j} \mathcal{P}_{m_{i}}^{M-1}$. Thus we need to show

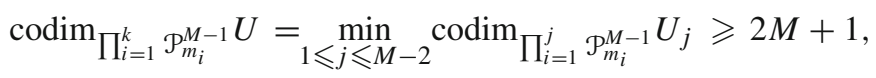

and this means to verify

$$
\operatorname{codim}_{\prod_{i=1}^{j} \mathcal{P}_{m_{i}}^{M-1}} U_{j} \geqslant 2 M+1, \quad 1 \leqslant j \leqslant M-2 .
$$

If $m_{j}=2$ (that is, $1 \leqslant j \leqslant k_{2}=k$ ) then we estimate the codimension of $U_{j}$ in $\prod_{i=1}^{j} \mathcal{P}_{m_{i}}^{M-1}$ using the method of [3]: Choose a general $(j-2)$-dimensional hyperplane $S \subset \mathbb{P}^{M-2}$. Then the projection $\pi_{S}: \mathbb{P}^{M-2} \rightarrow \mathbb{P}^{M-1-j}$ restricts to a finite morphism on each of irreducible components of $V\left(q_{1}, \ldots, q_{j-1}\right)$ for given $q_{1}, \ldots, q_{j-1}$ such that $\operatorname{codim}_{\mathbb{P}^{M-2}} V\left(q_{1}, \ldots, q_{j-1}\right)=j-1$. Consequently, polynomials in $\mathcal{P}_{m_{j}}^{M-1}=$ $\mathcal{P}_{2}^{M-1}$ obtained as a pullback of a homogeneous quadratic polynomial defined on $\mathbb{P}^{M-1-j}$ do not vanish on $V\left(q_{1}, \ldots, q_{j-1}\right)$. The linear space $W_{j}$ of such pulled-back quadratic polynomials has dimension $\left(\begin{array}{c}M-1-j+2 \\ 2\end{array}\right)$. By construction, $W_{j}$ intersects the space of polynomials in $\mathcal{P}_{2}^{M-1}$ vanishing on one of the irreducible components of $V\left(q_{1}, \ldots, q_{j-1}\right)$ only in 0 . Therefore, 
$\operatorname{codim}_{\prod_{i=1}^{j} \mathcal{P}_{m_{i}}^{M-1}} U_{j} \geqslant\left(\begin{array}{c}M-j+1 \\ 2\end{array}\right) \geqslant\left(\begin{array}{c}M-k+1 \\ 2\end{array}\right), \quad j=1, \ldots, k$.

Note that this estimate also holds if $j=1$. Furthermore, $\left(\begin{array}{c}M-k+1 \\ 2\end{array}\right) \geqslant 2 M+1$ since $3 k+4 \leqslant M$. Hence (6) is shown for $j=1, \ldots, k$.

If $m_{j}>2$ (that is, $k+1 \leqslant j \leqslant M-2$ ) then we estimate the codimension of $U_{j}$ in $\prod_{i=1}^{j} \mathcal{P}_{m_{i}}^{M-1}$ using the method of [4]: If $\left(q_{1}, \ldots, q_{j}\right) \in U_{j}$ choose an irreducible component $B$ of $V\left(q_{1}, \ldots, q_{j}\right)$. By definition of $U_{j}$, the codimension of $B$ in $\mathbb{P}^{M-2}$ is $j-1$. Assume that the codimension of the linear subspace $\langle B\rangle \subset \mathbb{P}^{M-2}$ spanned by $B$ is $b$; that means in particular that $0 \leqslant b \leqslant j-1$.

Lemma 4.1 If $b<j-1$, then there are indices $1 \leqslant i_{1}<\cdots<i_{j-1-b} \leqslant j-1$ such that $B$ is an irreducible component of $V\left(q_{i_{1}}, \ldots, q_{i_{j-1-b}}\right) \cap\langle B\rangle$.

Proof Since $\operatorname{codim}_{\mathbb{P}^{M-2}}\langle B\rangle=b<j-1=\operatorname{codim}_{\mathbb{P}^{M-2}} B$, one of $q_{1}, \ldots, q_{j-1}$ must not vanish on $\langle B\rangle$. Let $i_{1}$ be the smallest index such that $q_{i_{1} \mid\langle B\rangle} \not \equiv 0$. If $b=j-2$ one of the irreducible components of $V\left(q_{i_{1}}\right) \cap\langle B\rangle$ must be $B$ and we are done. For $b<j-2$ choose an irreducible component $R_{1}$ of $V\left(q_{i_{1}}\right) \cap\langle B\rangle$ containing $B$. Since $\operatorname{dim} R_{1}>\operatorname{dim} B$ and $q_{i \mid R_{1}} \equiv 0$ for $i=1, \ldots, i_{1}$, we can find an index $i_{1}+1 \leqslant i_{2} \leqslant$ $j-1$ such that $q_{i_{2}}$ does not vanish on $R_{1}$ : Otherwise, $R_{1} \subset V\left(q_{1}, \ldots, q_{j-1}\right)$, and this is a contradiction to $\operatorname{codim}_{\mathbb{P}^{M-2}} V\left(q_{1}, \ldots, q_{j-1}\right)=j-1$. In the same way we can inductively find $i_{3}, \ldots, i_{j-1-b}$ such that finally $B$ is an irreducible component of $V\left(q_{i_{1}}, \ldots, q_{i_{j-1-b}}\right) \cap\langle B\rangle$.

The lemma implies that $U_{j}$ is contained in the union of all locally Zariski-closed subsets $U_{j}\left(P ; i_{1}, \ldots, i_{j-1-b}\right) \subset \prod_{i=1}^{j} \mathcal{P}_{m_{i}}^{M-1}$ of the form

$$
\left\{\begin{array}{l}
\left.\left(q_{1}, \ldots, q_{j}\right): \begin{array}{l}
\text { there exists an irreducible component } B \text { of } \\
V\left(q_{i_{1}}, \ldots, q_{i_{j-1-b}}\right) \cap P \text { such that }\langle B\rangle=P \\
\operatorname{codim}_{P} B=j-1-b, q_{i \mid B} \equiv 0, i=1, \ldots, j
\end{array}\right\}
\end{array}\right.
$$

where $P$ ranges over all codimension $b$ linear subspaces of $\mathbb{P}^{M-2}, 0 \leqslant b \leqslant j-1$, and indices $i_{1}, \ldots, i_{j-1-b}$ range over all increasing sequences $1 \leqslant i_{1}<\cdots<i_{j-1-b} \leqslant$ $j-1$. Note that for $b=j-1$ we just consider the sets

$$
U_{j}(P)=\left\{\left(q_{1}, \ldots, q_{j}\right): \begin{array}{l}
P \text { is an irreducible component } \\
\text { of } V\left(q_{1}, \ldots, q_{j-1}\right) \text { and } q_{j \mid P} \equiv 0
\end{array}\right\} \subset \prod_{i=1}^{j} \mathcal{P}_{m_{i}}^{M-1}
$$

Since the dimension of the Grassmann variety $\mathbb{G}(M-2-b, M-2)$ is $b(M-1-b)$, estimate (6) will follow if

$$
\operatorname{codim}_{\prod_{i=1}^{j} \mathcal{P}_{m_{i}}^{M-1}} U_{j}\left(P ; i_{1}, \ldots, i_{j-1-b}\right) \geqslant 2 M+1+b(M-1-b)
$$

holds. By a linear change of coordinates we can assume that

$$
P=\left\{z_{M-b}=\cdots=z_{M-1}\right\}
$$


Let $q_{i_{1}}, \ldots, q_{i_{j-1-b}}$ be polynomials in $\prod_{r=1}^{j-1-b} \mathcal{P}_{m_{i_{r}}}^{M-1}$ such that an irreducible component $B$ of $V\left(q_{i_{1}}, \ldots, q_{i_{j-1-b}}\right)$ lies in $P$ with $\operatorname{codim}_{P} B=j-1-b$ and $\langle B\rangle=P$. Then for any degree $m$, products of $m$ linear forms

$$
\prod_{i=1}^{m}\left(a_{i, 1} z_{1}+\cdots+a_{i, M-b-1} z_{M-b-1}\right)
$$

do not vanish on $B$. These products span a linear subspace of $\mathcal{P}_{m}^{M-1}$ of dimension $(M-b-2) m+1$ intersecting the linear subspace of polynomials vanishing on $B$ only in 0 . We apply these facts to polynomials $q_{i} \in \mathcal{P}_{m_{i}}^{M-1}$ with $i \in\{1, \ldots, j\}-$ $\left\{i_{1}, \ldots, i_{j-1-b}\right\}$ and obtain

$$
\begin{aligned}
\operatorname{codim}_{\prod_{i=1}^{j} \mathcal{P}_{m_{i}}^{M-1}} U_{j}\left(P ; i_{1}, \ldots, i_{j-1-b}\right) & \geqslant(M-b-2) \sum_{\substack{i \notin\left\{i_{1}, \ldots, i_{j-b-1}\right\} \\
i}}^{j} m_{i}+b+1 \\
& \geqslant\left(\sum_{i=1}^{b} m_{i}+m_{j}\right)(M-b-2)+b+1 .
\end{aligned}
$$

So (8) follows from

$$
\left(\sum_{i=1}^{b} m_{i}+m_{j}-b\right)(M-b-2) \geqslant 2 M .
$$

Since $m_{i} \geqslant 2$ for $i=1, \ldots, j-1$ and we consider the case $m_{j} \geqslant 3$, we have

$$
\sum_{i=1}^{b} m_{i}+m_{j}-b \geqslant b+3 .
$$

The polynomial $(b+3)(M-2-b)-2 M=-b^{2}+(M-5) b+M-6$ quadratic in $b$ increases for $b \leqslant(M-5) / 2$ and decreases for $b \geqslant(M-5) / 2$. Since $M \geqslant 3 k+4 \geqslant 7$, hence $3(M-2)-2 M \geqslant 0$, estimate (9) is shown for $b=0, M-5$, hence for all $0 \leqslant b \leqslant M-5$. This leaves to consider the cases $b=M-4, M-3$.

If $b=M-4$ then $j=M-3$ or $M-2$, and by the assumptions on the degrees $m_{i}$ we have

$$
\begin{aligned}
\sum_{i=1}^{b} m_{i}+m_{j} & \geqslant \sum_{i=1}^{M} m_{i}-2 d_{k}-\left(d_{k}-1\right) \\
& =\sum_{l=1}^{k-1} \frac{d_{l}\left(d_{l}+1\right)}{2}+\frac{\left(d_{k}-3\right)\left(d_{k}-2\right)}{2}+2-k
\end{aligned}
$$


Similarly, if $b=M-3$ then $j=M-2$, and

$$
\begin{aligned}
\sum_{i=1}^{b} m_{i}+m_{j} & \geqslant \sum_{i=1}^{M} m_{i}-d_{k}-d_{k} \\
& =\sum_{l=1}^{k-1} \frac{d_{l}\left(d_{l}+1\right)}{2}+\frac{\left(d_{k}-2\right)\left(d_{k}-1\right)}{2}+1-k
\end{aligned}
$$

This last case is the worst possible situation: $V\left(q_{1}, \ldots, q_{M-3}\right)$ is a line in $\mathbb{P}^{M-2}$, and $q_{M-2}$ vanishes on this line.

Solving an optimization problem and using $\sum_{l=1}^{k} d_{l}=M+k$ we obtain

$$
\begin{aligned}
& \sum_{l=1}^{k-1} d_{l}^{2}+\left(d_{k}-3\right)^{2} \geqslant k\left(\frac{M-3+k}{k}\right)^{2} \quad \text { and } \\
& \sum_{l=1}^{k-1} d_{l}^{2}+\left(d_{k}-2\right)^{2} \geqslant k\left(\frac{M-2+k}{k}\right)^{2} .
\end{aligned}
$$

Consequently, (9) follows for $b=M-4, M-3$ if

$$
\left[\frac{(M-3+k)^{2}}{2 k}+\frac{M-3+k}{2}-k-M+6\right] \cdot 2 \geqslant 2 M
$$

and

$$
\left[\frac{(M-2+k)^{2}}{2 k}+\frac{M-2+k}{2}-k-M+3\right] \cdot 1 \geqslant 2 M .
$$

Now (10) is equivalent to

$$
k \leqslant \frac{(M-3)^{2}}{M}
$$

and (11) is equivalent to

$$
k \leqslant \frac{(M-2)^{2}}{3 M-2} \text {. }
$$

Both inequalities are satisfied if $M \geqslant 3 k+4$. Then $k \geqslant 1$ implies $M \geqslant 7$, so no further lower bound on $M$ is needed. Proof of Theorem 3.2 is complete.

\section{Hypertangent divisors}

Let us prove Theorem 2.3. The argument is similar to the proof of [6, Theorem 4], so we will only sketch the main steps.

Step 1. Assume that the pair $(V, D / n)$ is not canonical for an effective divisor $D \sim$ $n H$, where $H \in$ Pic $V$ is the class of a hyperplane section, $K_{V}=-H$. By linearity of all conditions involved in this assumption, the divisor $D$ can be assumed to be 
irreducible. For some prime divisor $E$ over $V$ the inequality $\operatorname{ord}_{E} D>n a(E, V)$ holds.

We look at the centre $B \subset V$ of the divisor $E$. Since by [6, Proposition 3.6] for any irreducible subvariety $Y \subset V$ of dimension at least $k$ (where $k=\operatorname{codim}(V \subset \mathbb{P})$ ) we have mult $_{Y} D \leqslant n$, we conclude that $\operatorname{dim} B \leqslant k-1$. Let $o \in B$ be a point of general position, $\sigma: V^{+} \rightarrow V$ its blow up and $E^{+}=\sigma^{-1}(o)$ the exceptional divisor. A standard argument (see, for example, [6, Proposition 2.5]) gives us a hyperplane $\Delta \subset E^{+} \cong \mathbb{P}^{M-1}$ satisfying the inequality

$$
\operatorname{mult}_{o} D+\text { mult }_{\Delta} D^{+}>2 n,
$$

where $D^{+}$is the strict transform of $D$ on $V^{+}$.

Now let $T$ be a general hyperplane section of $V$, containing the point $o$ and cutting out $\Delta$ on $E^{+}$: that is to say, $T^{+} \cap E^{+}=\Delta$. It is easy to see that the effective cycle of the scheme-theoretic intersection $D_{T}=(D \circ T)$ is well defined and satisfies the estimate

$$
\operatorname{mult}_{o} D_{T}>2 n \text {. }
$$

We will consider $D_{T}$ as an effective divisor on the complete intersection $T \subset \mathbb{P}^{M+k-1}$ of codimension $k, D_{T} \sim n H_{T}$.

Step 2. By Sects. 3-4, the complete intersection $T \subset \mathbb{P}^{M+k-1}$ satisfies the regularity condition. Namely, for a system of linear coordinates $z_{1}, \ldots, z_{M+k-1}$ with the origin at the point $o$, the variety $T$ is given by a system of non-homogeneous polynomial equations:

$$
\begin{gathered}
\bar{f}_{1}=\bar{q}_{1,1}+\cdots+\bar{q}_{1, d_{1}}, \\
\cdots \\
\bar{f}_{i}=\bar{q}_{i, 1}+\cdots+\bar{q}_{i, d_{i}}, \\
\cdots \\
\bar{f}_{k}=\bar{q}_{k, 1}+\cdots+\bar{q}_{k, d_{k}},
\end{gathered}
$$

where the bar means the restriction onto the hyperplane $\{l=0\}-$ the hyperplane that cuts out $T$ on $V$. Now the set of homogeneous polynomials

$$
\left\{\bar{q}_{i, j}:(i, j) \in I\right\}
$$

forms a regular sequence in $\mathcal{O}_{o, \mathbb{P}^{M+k-1}}$.

Step 3. Now we can apply the technique of hypertangent divisors [8, Chapter 3] to the divisor $D_{T}$ on the complete intersection $T \subset \mathbb{P}^{M+k-1}$ in precisely the same way as it was done in [6, Section 3] or, in more details in [7, Section 5], and obtain the estimate

$$
\frac{\operatorname{mult}_{o} D_{T}}{2 n} \leqslant \max \left\{1, \frac{3}{4} \cdot \frac{d_{k}}{d_{k}-1} \cdot \frac{d_{+}}{d_{+}-1}\right\},
$$


where $d_{+}=d_{k}$, if $d_{k-1}=d_{k}$, and $d_{+}=d_{k}-1$, otherwise. It is easy to see that if $d_{k} \geqslant 8$, this gives us the inequality mult ${ }_{o} D_{T} \leqslant 2 n$, which contradicts the estimate (14) obtained in Step 1. The contradiction completes the proof of Theorem 2.3.

Proof of Theorem 2.4 It follows the same lines and repeats verbatim the argument given in [7, Sect. 4]. What is different from the proof of Theorem 2.3 given above, is the starting point: assuming the inequality

$$
\operatorname{ct}(V) \leqslant \frac{M}{M+1},
$$

we obtain for any rational number $\lambda>M /(M+1)$ an effective divisor $D \sim n H$ such that the pair $(V, \lambda D / n)$ is not canonical. Now, repeating the proof of Theorem 2.3, we use the technique of hypertangent divisors to obtain the inequality

$$
1<\lambda \max \left\{1, \frac{3}{4} \cdot \frac{d_{k}}{d_{k}-1} \cdot \frac{d_{+}}{d_{+}-1}\right\},
$$

and taking the limit as $\lambda \rightarrow M /(M+1)$, we conclude that

$$
\max \left\{1, \frac{3}{4} \cdot \frac{d_{k}}{d_{k}-1} \cdot \frac{d_{+}}{d_{+}-1}\right\} \geqslant \frac{M+1}{M} .
$$

However, it is a trivial check that in the assumptions of Theorem 2.4 the last inequality cannot be true.

\section{References}

1. Demailly, J.-P., Kollár, J.: Semi-continuity of complex singularity exponents and Kähler-Einstein metrics on Fano orbifolds. Ann. Sci. École Norm. Sup. 34(4), 525-556 (2001)

2. Nadel, A.M.: Multiplier ideal sheaves and Kähler-Einstein metrics of positive scalar curvature. Ann. Math. 132(3), 549-596 (1990)

3. Pukhlikov, A.V.: Birational automorphisms of Fano hypersurfaces. Invent. Math. 134(2), 401-426(1998)

4. Pukhlikov, A.V.: Birationally rigid Fano complete intersections. J. Reine Angew. Math. 541, 55-79 (2001)

5. Pukhlikov, A.V.: Birational geometry of Fano direct products. Izv. Math. 69(6), 1225-1255 (2005)

6. Pukhlikov, A.V.: Birational geometry of algebraic varieties with a pencil of Fano complete intersections. Manuscripta Math. 121(4), 491-526 (2006)

7. Pukhlikov, A.V.: Existence of the Kähler-Einstein metric on certain Fano complete intersections. Math. Notes 88(3-4), 552-558 (2010)

8. Pukhlikov, A.: Birationally Rigid Varieties. Mathematical Surveys and Monographs, vol. 190. American Mathematical Society, Providence (2013)

9. Tian, G.: On Kähler-Einstein metrics on certain Kähler manifolds with $C_{1}(M)>0$. Invent. Math. 89(2), 225-246 (1987) 